\title{
44396 - POSTOPERATIVE COMPLICATIONS AMONG OBSTRUCTIVE SLEEP APNEA PATIENTS
}

\author{
Balaji Yegneswaran, University of Toronto, University Health Network, Toronto, ON, \\ Canada; \\ Balaji Yegneswaran, University of Toronto, University Health Network; \\ Peter Liao, University of Toronto, University Health Network; \\ $S$ Vairavanathan, University of Toronto, University Health Network; \\ A Zaki, University of Toronto, University Health Network; \\ F Chung, University of Toronto, University Health Network;
}

Introduction: Obstructive Sleep Apnea (OSA) is presumed to be a risk factor for perioperative morbidity and mortality. At present the data quantifying the magnitude of perioperative complications due to OSA is very limited to make any major correlations. This retrospective study is designed to document the occurrence of perioperative respiratory complications among surgical patients with OSA.

Methods: Hospital ethics approval was obtained. All OSA patients age $>18$ years who underwent surgeries other than uvulopalatopharyngoplasty in 3 hospitals of University Health Network for 15 years from 1990 to 2005 were selected. Chart review and data analysis was focused on respiratory complications which included desaturation (mild: SaO2\&\#8804;95\%, severe: $\mathrm{SaO} 2 \& \# 8804 ; 90 \%$ ), pulmonary edema, bronchospasm and re-intubation; cardiac and neurological complications. The treatments for the complications were also documented. Result: 262 charts were reviewed: age: $56 \pm 13$ years; male: $74.4 \%$; female: $25.6 \%$; BMI: $35.1 \pm$ $9.6 \mathrm{~kg} / \mathrm{m} 2$ and ASA status I: $1.9 \%$, II: $32.7 \%$, III: $51.9 \%$, IV: $13.5 \%$. The major pre-existing diseases were: hypertension (49.2\%), GERD (26.3\%), coronary artery disease $(27.5 \%)$ and diabetes (22.1\%). 64.5\% OSA patients were on home CPAP. The total incidence of postoperative complication was $42.8 \%(n=112)$. The incidence of respiratory complications was $32.8 \%(\mathrm{n}=86)$. The most common was desaturation which was seen in $78(29.8 \%)$ patients (mild: $n=35$, severe: $n=43)$. Other complications were pulmonary edema $(3.1 \%)$, bronchospasm $(2.3 \%)$, and pneumothorax $(0.8 \%)$. As a result of the complications, $22.9 \%$ patients needed prolonged oxygen therapy during their postoperative period; $27.5 \%$ patients who had not been on home CPAP required CPAP postoperatively; 5 patients had unplanned ICU admission of whom 1 patient had to be intubated and 2 patients had episodes of hypercapnea.

The incidence of cardiac complication was $14.5 \%(\mathrm{n}=38)$, including arrhythmia (12.6\%), cardiac arrest $(1.2 \%)$ and ischemia $(0.8 \%)$. The incidence of neurological complications was $6.1 \%$ $(\mathrm{n}=16)$ : agitation $(3.4 \%)$, confusion $(1.5 \%)$, motor deficit $(1.2 \%)$ and sensory deficit $(1.2 \%)$. Discussion: OSA surgical patients had a high incidence of postoperative complications (42.8\%). Respiratory complications was most common (32\%) and extra treatment was often required. 
Demographic and Complication Data of OSA Patients (n-262)

Age (year)

BMI $\left(\mathrm{kg} / \mathrm{m}^{2}\right)$

Gender

Male

Female

Pre-Existing Medical Conditions

Hypertension

GERD

Diabetes

Postoperative Complication

Respiratory Complication

Total Desaturation $(\mathrm{SaO} 2 \leq 95 \%)$

Mild Desaturation (SaO2: 90 95\%)

Severe Desaturation $(\mathrm{SaO} 2 \leq 90 \%)$

Cardiac Complication

Arrhythmia

Cardiac Arrest

Ischemia

Neurology Complication

Agitation

Confusion

Motor Deficit

Sensory Deficit
Mean \pm SD

$55.5 \pm 13.2$

$35.1 \pm 9.6$

Frequency (\%)

$195(74.4)$

67 (25.6)

129(49.2)

69(26.3)

$58(22.1)$

$112(42.8)$

$86(32.8)$

78 (29.8)

35 (13.4)

43 (16.4)

38 (14.5)

33 (12.6)

$3(1.15)$

$2(0.76)$

$16(6.11)$

9(3.44)

4 (1.5)

$3(1.15)$

$3(1.15)$ 